\title{
Forward genetic approaches for elucidation of novel regulators of Lyme arthritis severity
}

\author{
Kenneth K.C. Bramwell ${ }^{1}$, Cory Teuscher ${ }^{2}$ and Janis J. Weis ${ }^{1 *}$ \\ ${ }^{1}$ Department of Pathology, University of Utah, Salt Lake City, UT, USA \\ ${ }^{2}$ Department of Medicine, University of Vermont, Burlington, VT, USA
}

\section{Edited by:}

Tanja Petnicki-Ocwieja, Tufts

University School of Medicine and

Tufts Medical Center, USA

Reviewed by:

Janakiram Seshu, The University of

Texas at San Antonio, USA

Philip E. Stewart, National Institute

of Allergy and Infectious Disease,

USA

*Correspondence:

Janis J. Weis, Department of

Pathology, University of Utah, 15

North Medical Drive East \#2100,

Salt Lake City, UT 84112-5650, USA

e-mail: janis.weis@path.utah.edu
Patients experiencing natural infection with Borrelia burgdorferi display a spectrum of associated symptoms and severity, strongly implicating the impact of genetically determined host factors in the pathogenesis of Lyme disease. Herein, we provide a summary of the host genetic factors that have been demonstrated to influence the severity and chronicity of Lyme arthritis symptoms, and a review of the resources available, current progress, and added value of a forward genetic approach for identification of novel genetic regulators.

Keywords: Lyme disease, Lyme arthritis, forward genetics, pathogenesis, genome-wide association studies, innate immunity and responses, inflammation, beta-Glucuronidase

\section{INTRODUCTION TO LYME DISEASE}

Lyme Disease, caused by infection with the tick borne spirochete Borrelia burgdorferi, is a growing societal concern, especially in endemic regions of the United States and Europe. Approximately 30,000 case reports are filed by physicians each year in the United States (C.D.C, 2013a), while the CDC has upwardly revised their best estimate of the total incidence to $300,000 /$ year, based on several complementary lines of evidence (Kuehn, 2013). Part of the societal concern is rooted in the uncertainty surrounding pathological outcomes associated with B. burgdorferi infection. A large percentage $(70 \%)$ of infected individuals develop the characteristic bulls-eye rash erythema migrans at the site of the infected tick bite, with progression to further clinical complications following dissemination of the spirochete. Arthritis, the most common symptom occurs in $30-60 \%$ of infected individuals, while Bell's palsy and other neurological symptoms are seen in $10-12 \%$ of patients (Wormser et al., 2006; C.D.C, 2013b). Carditis has been considered a rare complication $(<1 \%)$, however, 3 recent deaths with documented B. burgdorferi in autopsied heart tissue strongly argue for increased vigilance in detecting infection of this tissue (C.D.C, 2013c).

The wide variation in Lyme disease symptoms and severity observed within the patient population is thought to reflect unique features of individual $B$. burgdorferi isolates that influence invasive potential, as well as heritable factors in the patient population that contribute to clinical severity. Furthermore, although most patients resolve infection with appropriate antibiotic therapy, a small percentage of treated patients with severe clinical symptoms fail to resolve and develop a chronic disease termed Post Treatment Lyme Disease (Steere and Glickstein, 2004). Thus, there are compelling reasons to identify host genes that determine the severity of Lyme disease, both in understanding the pathogenic mechanisms of acute clinical disease and in characterizing predisposing features for chronic disease. This review will assess past and ongoing studies that have provided insight into genetic susceptibility to Lyme arthritis, with particular emphasis on studies using Forward Genetic approaches.

\section{WHAT IS FORWARD GENETICS?}

Forward genetics is an unbiased genetic approach that begins with a heritable trait of interest and attempts to determine the alleles responsible for the observed variability through a process of genetic mapping. In contrast, reverse genetics is a hypothesisdriven scientific approach that begins with a gene of interest and attempts to determine the phenotypic impacts caused by experimental manipulations of that gene. Classically, forward genetic studies were first performed through random mutagenesis screens. More recently, forward genetics has been used to map pre-existing genetic variations in human populations or experimental animal models. In practice, forward genetic studies involve three key steps: (1) Each individual in a population of mixed genetic composition is surveyed for the trait of interest; (2) The genetic makeup of each individual is assessed; (3) Statistical calculations predict the strength of association in the study population between the measured trait and each genetic locus in the genome. Forward genetic screens often produce a map across the entire genome with peaks and valleys denoting areas with strong or weak statistical association with the trait, respectively.

Scientific investigations into heritable genetic risk factors that contribute to complex disease have been conducted using a variety of approaches, including Genome Wide Association Studies (GWAS) and forward genetic screens in tractable animal models. 


\section{GENOME WIDE ASSOCIATION STUDIES AS A METHODOLOGY FOR STUDYING COMPLEX GENETIC TRAITS IN HUMANS - SUCCESSES AND LIMITATIONS}

Genome Wide Association Studies were first proposed in the mid1990 s as a way to study association between human genetic polymorphisms and complex, multigenic traits. Rather than measure all genetic variation present in each individual, approximately one million Single Nucleotide Polymorphisms (SNPs) are assayed as genetic landmarks, and scored for association with the trait of interest. Based on linkage disequilibrium, genes in close proximity to associated SNP landmarks are potential candidates for further investigation.

This technique is well-suited to identify susceptibility genes that are of intermediate prevalence in the population, with a Mean Allele Frequency of greater than 0.05 (Risch and Merikangas, 1996). Although no such studies of Lyme arthritis severity have been conducted, GWAS has been extensively used to investigate genetic modulators of other inflammatory conditions including rheumatoid arthritis (RA). Thus far, almost fifty susceptibility loci have been identified for RA, accounting for approximately one-half of the total genetic variation expected in populations of European ancestry. Nineteen of these loci have been refined to a single candidate gene association, and the underlying causal polymorphism has been predicted for seven of these loci (Eyre et al., 2012).

Recent studies with RA and juvenile RA have involved cohorts of up to 10,000 patients and controls, pointing out the requirement for large populations of well-characterized patients for GWAS analysis (Hinks et al., 2013). If sufficiently large sample sizes could be achieved, these findings suggest that GWAS could be a successful strategy to investigate Lyme arthritis susceptibility loci, but also indicate that additional approaches are needed to capture the significant fraction of variation likely to be left unaccounted for.

It is also important to recognize that identification of regulatory loci is not the ultimate goal of a forward genetics study, but is only a first step. While association of a specific genetic landmark to disease susceptibility may have potential relevance to clinical diagnosis, there is added value in the formal investigation of candidate genes and predicted causal polymorphisms, and in further understanding the underlying mechanisms of pathogenesis. This type of mechanistic investigation frequently involves the use of animal models.

\section{USE OF ANIMAL MODELS FOR IDENTIFICATION OF GENES REGULATING DISEASE SEVERITY}

Animal models provide an alternative approach for identification of genes that regulate disease development. Inbred mouse lines are powerful genetic resources, which have been widely used to identify genes associated with disease severity. Visionary scientists began the breeding of inbred mice over a century ago. Each modern inbred line has fixed genetic composition, while the plentitude of inbred strains collectively capture a large amount of genetic variation. Several advances in the past decade have significantly added to their value, particularly the publication of the mouse genome, coupled with various efforts to define the genetic variation between inbred mouse strains (Gregory et al., 2002; Keane et al., 2011). More recently, the Collaborative Cross was developed, which represents an ambitious community effort by mouse geneticists to develop approximately 1000 additional recombinant inbred mouse strains with defined genetic composition. These recombinant mice were derived from 8 parental inbred and wild-derived strains through an intricate directed breeding process (Churchill et al., 2004). Due to the increased genetic diversity of mouse strains that can be interrogated simultaneously, the Collaborative Cross is expected to provide additional power to forward genetics screens for many diseases. Together, these more recent developments are expected to provide greater predictive power for identification of regulatory intervals underlying complex multigenic traits.

Prior to the availability of these more sophisticated modern resources, a seminal study by Dr. Stephen Barthold recognized that inbred mouse strains exhibit distinct genetic susceptibilities to Lyme arthritis, recapitulating the range of arthritis severity seen in patients (Barthold et al., 1990). However, mice do not recapitulate the full depth and breadth of symptoms experienced by human patients. This is evident in the inability of B. burgdorferi to elicit neurological symptoms or erythema migrans in mice (Garcia-Monco and Benach, 2013). Despite these limitations, the finding that $\mathrm{C} 3 \mathrm{H}$ mice develop severe arthritis and carditis at reproducible times following intradermal infection with B. burgdorferi cultured in the laboratory was very important. The $\mathrm{C} 3 \mathrm{H}$ mouse has been used extensively for studies of severe disease, and the involvement of a variety of cell types and signaling pathways have been evaluated. Equally important was the observation that C57BL/6 (B6) mice consistently develop less severe disease despite being equally susceptible to infection and having similar numbers of bacteria in joints. Since many mutant alleles have been crossed onto the B6 background, this has allowed identification of the contribution of numerous immunologically important genes to both host defense and modulation of arthritis and carditis severity.

Mice and other small mammals are essential reservoir species for B. burgdorferi in nature, and greater than $90 \%$ of trapped wild mice in some Lyme endemic areas have tested seropositive for infection (Bunikis et al., 2004; Radolf et al., 2012). Wild mice are generally resistant to Lyme arthritis, although recent work has shown that natural variants of known innate immune regulatory genes may be correlated with the prevalence of Lyme infection within the wild rodent population (Tschirren et al., 2013). These considerations make the mouse an excellent model for assessment of genetic factors contributing to Lyme arthritis development.

Barthold's findings were corroborated and expanded upon by others. Many groups then addressed specific facets of the immune response and pathogenesis of Lyme arthritis, using the mouse models developed by Barthold, and that important work continues today. Many such studies rely on reverse-genetic approaches, such as targeted genetic deletion, gene silencing, treatment with inhibitory or stimulatory molecules, or transgenic manipulation. For example, the importance of the innate immune response in Lyme pathogenesis was demonstrated by Schaible, Barthold, and Brown who collectively observed that mice with severe combined immunodeficiency (scid/Rag ${ }^{-}$), lacking $\mathrm{B}$ and $\mathrm{T}$ cells, 
retained the differential genetic severities in arthritis and carditis observed between inbred mouse strains (Schaible et al., 1989; Barthold et al., 1992; Brown and Reiner, 1999). This finding set a lasting framework for future studies into various facets of the innate immune response. The reverse genetic techniques used in these and other studies are powerful and conclusive, and have resulted in the identification of many genes with documented importance in the pathogenesis of Lyme arthritis and host defense to $B$. burgdorferi. However, these approaches are by nature biased to genes with known function and are not suitable for global analysis of the potential genetic contribution to disease. Selection of a candidate gene necessarily involves assessment of pathways that are suspected to influence the disease process, resulting in the rejection or delay of other non-candidate genes for study.

\section{BRIEF REVIEW OF GENETIC ASSOCIATIONS WITH HUMAN LYME DISEASE AND RELATED MOUSE STUDIES ROLE OF THE MHC IN IMMUNE RESPONSE TO B. BURGDORFERI AND IN ARTHRITIS SEVERITY: HUMAN AND MOUSE STUDIES}

Several important studies have discovered natural genetic alleles that influence Lyme arthritis severity. Steere et al. first reported the influence of the human major histocompatibility complex (MHC) on Lyme arthritis severity, and provided important early evidence that Lyme arthritis has an immunogenetic basis (Steere et al., 1990). This pioneering work identified increased incidence of clinical Lyme arthritis, particularly that lasting longer that 12 months in a single joint, as associated with two serologically defined Class II alleles, HLA-DR4 and HLA-DR2. Importantly, the association of Class II alleles with Lyme arthritis was not supported by studies inclusive of all outcomes of Lyme arthritis patients. The advent of molecular characterization of Class II alleles allowed more precise analysis of associations with disease phenotype, and led to the conclusion that MHC alleles are not major determinants of early Lyme disease severity, a distinction from rheumatoid arthritis (Feng et al., 1995; Klempner et al., 2005). More recently, Steere and colleagues have confirmed the association of two Class II alleles (DRB1*0101 and 0401) for the subgroup of patients with treatment refractory Lyme disease but not in the larger group of patients that respond to antibiotic treatment, and have proposed an auto-immune mechanism in this treatment refractory group (Steere et al., 2006; Drouin et al., 2013).

A number of investigators found association of $\mathrm{MHC}$ haplotypes with antibody recognition of individual B. burgdorferi antigens using MHC congenic mouse lines. However, use of MHC congenics in our studies and in those of other investigators led to the conclusion that MHC alleles were not determinants for the differences in arthritis severity found 4 weeks following infection in $\mathrm{C} 3 \mathrm{H}-\mathrm{H} 2^{k}, \mathrm{C} 57 \mathrm{BL} / 6-\mathrm{H} 2^{b}$, and DBA-H2 $2^{d}$ mice (Yang et al., 1992; Brown and Reiner, 2000). Thus, studies with mice are consistent with patient studies failing to show association with early Lyme arthritis. Interestingly, mice expressing the $H 2^{k}$ allele do not develop collagen-induced arthritis, a contrast with their development of severe Lyme arthritis (Wooley et al., 1981).

\section{IDENTIFICATION OF TLR1/TLR2 IN THE HOST RESPONSE TO B. BURGDORFERI IN HUMANS AND MICE}

Early seminal studies into the host-pathogen interaction of $B$. burgdorferi revealed the potential of the spirochete and its lipoproteins to induce inflammatory cytokine production in a variety of human and mouse cell types (Radolf et al., 1991; Wooten et al., 1996; Sellati et al., 1998). The association of $\mathrm{NF}-\kappa \mathrm{B}$ with these inflammatory responses directed numerous laboratories to investigate the involvement of Toll-like receptors as these molecules were discovered as central components of inflammatory responses to microbial pathogens (Wooten et al., 1996; Sellati et al., 1998). These studies documented the interactions between B. burgdorferi lipoproteins with TLR2 and TLR1, both with mouse knock-out and cell culture transfection studies and in patients, and established a critical role for TLR signaling through MyD88 in host defense to this pathogen (Aliprantis et al., 1999; Brightbill et al., 1999; Hirschfeld et al., 1999, 2000; Alexopoulou et al., 2002). More recent studies by Schroder et al. identified a human variant in TLR2, Arg753Gln, with reduced pro-inflammatory signaling in patient samples (Schroder et al., 2005). Cells from mice heterozygous for this variant also displayed reduced inflammatory responses to B. burgdorferi lysate. Notably, this TLR2 allele was significantly underrepresented within a cohort of late stage Lyme disease patients, suggesting that it has a protective effect.

Oosting et al. found that N248S and S602I polymorphisms in TLR1 were associated with reduced in vitro responsiveness to B. burgdorferi and TLR1/TLR2 agonist stimulation (Oosting et al., 2011a). Using a similar experimental approach, the same group also reported that peripheral blood mononuclear cells (PBMCs) for individuals bearing an IL-23R Arg381Gln polymorphism exhibited a reduced Th17 response following in vitro stimulation with B. burgdorferi (Oosting et al., 2011b). However, there was no association between the IL-23R polymorphism and the persistence of symptoms among patients in the study population, arguing against a role for this SNP in disease pathogenesis.

Strle et al. recently described the frequency and impact of several polymorphisms in the TLR1 gene within a cohort of Lyme disease patients (Strle et al., 2012). This study found a skewed inheritance pattern of TLR1 1805GG polymorphisms within an antibiotic-refractory Lyme arthritis patient population. They also recognized a synergy between inheritance of this host polymorphism and infection with a particular invasive isolate (termed RST1) of B. burgdorferi. Importantly, patients carrying TLR1 1805GG exhibited higher serum levels of CXCL9 and CXCL10 chemokines, consistent with a functional role for this polymorphism. This effect was reproduced through in vitro activation of PBMCs with a $B$. burgdorferi RST1 isolate, arguing that heightened production of these IFN $\gamma$-inducible chemokines may set the stage for antibiotic refractory arthritis.

\section{THE POWER OF FORWARD GENETICS}

Forward Genetic approaches attempt to determine which genetic loci are responsible for a phenotype of interest. In general, individuals are generated with genotypes that have been altered in an unbiased way, followed by analysis to map inheritance of the phenotype of interest to specific genetic loci. This was made possible 
by the development of genetic maps of microsatellite landmarks evenly distributed throughout the genome, the utility of which Paterson et al. first demonstrated for Quantitative Trait Locus (QTL) mapping in plants, later followed by Todd et al. in mice (Paterson et al., 1988; Todd et al., 1991). Model organisms are often studied through QTL analysis followed by the breeding of recombinant inbred congenic lines to isolate regulatory loci, and the Collaborative Cross is a more expansive modern variation of this theme that combines these two steps together. QTL mapping of disease susceptibility in mice has the potential to yield a veritable avalanche of information about complementary facets of disease initiation and pathogenesis. For example, efforts by Edward Wakeland and others to determine differential susceptibility to systemic lupus erythematosus (sle) between resistant and acutely lupus-prone inbred mouse strains led to the identification of Ly108 and other SLAM family members as key modulators of B cell tolerance (Kumar et al., 2006), lack of proper Fcgr2b upregulation as a potentiator of IgG production (Rahman et al., 2007), Cr2 or other closely linked genes as mediators of autoreactive B- and T-cell production (Chen et al., 2005; Tchepeleva et al., 2010), and hemostatic kallikreins as important regulators of kidney pathogenesis (Liu et al., 2009).

Other successes include the identification of genes important in the regulation of animal models of rheumatoid arthritis and autoimmunity by comparing disease susceptible and disease resistant mouse strains (Ma et al., 2002; Glant et al., 2004; Wicker et al., 2005; Ahlqvist et al., 2009). In some cases, QTL mapping efforts have bridged gaps between seemingly distinct experimental models of autoimmune and other inflammatory diseases through the identification of shared immunopathology loci (Teuscher, 1985; Meeker et al., 1995; Teuscher et al., 1996, 1997, 1998; Del Rio et al., 2008; Spach et al., 2009, 2010) and identification of the relevant functional polymorphisms (Sudweeks et al., 1993; Ma et al., 2002).

The first step of QTL analysis in mice is the direct interbreeding of two strains of interest to generate a large cohort of genetically distinct individuals (Figure 1). $\mathrm{F}_{1}$ hybrids are genetically identical, carrying one copy of each chromosome from each parental line. These hybrids can be backcrossed to either parental strain (BC1), or interbred to generate $F_{2}$ hybrids. In each case, genetic variability among the offspring is generated by random recombination events between sister chromatids during meiosis. Each interbreeding strategy can identify regulatory alleles with a dominant, codominant, or additive effect. $\mathrm{F}_{2}$ intercross populations allow the identification of alleles acting in recessive fashion that are capable of "standing alone," whereas the $\mathrm{BC} 1$ populations have the added advantage of allowing identification of genetic alleles whose effect is most apparent in the genetic context of a particular inbred background. However, hybrids backcrossed to a parental strain are not expected to detect any phenotype from recessive alleles bred back to a dominant parent.

\section{CURRENT PROGRESS: MAPPING QUANTITATIVE TRAIT LOCI THAT REGULATE LYME ARTHRITIS SEVERITY IN MICE}

Janis Weis, Cory Teuscher, and their collaborators performed the first murine Lyme arthritis QTL analysis (Weis et al., 1999). This initial study used an $\mathrm{F}_{2}$ intercross between C57BL/6N (B6) and

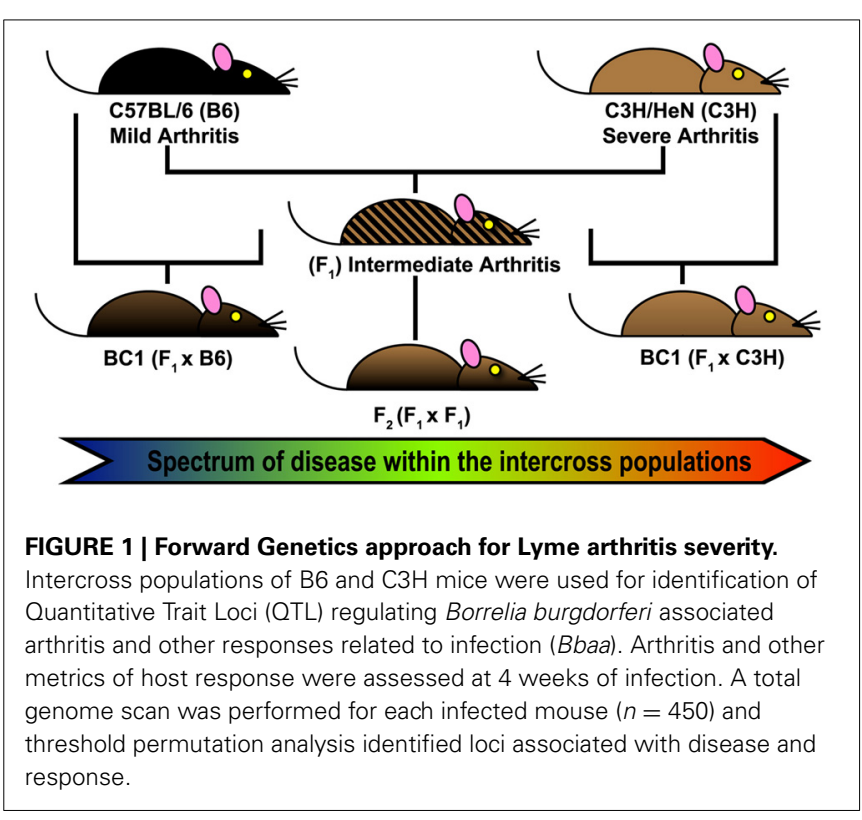

$\mathrm{C} 3 \mathrm{H} / \mathrm{HeN}(\mathrm{C} 3 \mathrm{H})$ mice, with 150 total male and female mice included in the cohort. Each individual was assessed for seven quantitative traits and for genetic composition, which was determined using 195 microsatellite markers distributed throughout the genome. Permutation threshold analysis was then used for the entire cohort to determine the degree of association between these quantitative traits and the parental derivation of specific loci. Four distinct regions on chromosomes 4, 5, and 11 were found to regulate arthritis severity traits as measured by caliper measurement of ankle swelling and by blinded scoring of a number of microscopically assessed histopathology traits. Five additional loci on chromosomes $6,9,11,12$, and 17 were found to regulate $B$. burgdorferi-specific humoral $\operatorname{IgM}$ and $\operatorname{IgG}$ responses independently of arthritis severity.

This foundational study was followed up by Roper et al. with additional QTL experiments using reciprocal $\mathrm{F}_{1} \times \mathrm{B} 6$ and $\mathrm{F}_{1} \times$ $\mathrm{C} 3 \mathrm{H}$ backcrosses and a $(\mathrm{BALB} / \mathrm{c} \times \mathrm{C} 3 \mathrm{H}) \mathrm{F}_{1} \times \mathrm{C} 3 \mathrm{H}$ intercross that found 12 new QTL on Chromosomes 1, 2, 4, 6, 7, 9, 10, $12,14,15,16$, and 17 regulating a variety of traits (Roper et al., 2001). A total of twenty-three QTL were identified that regulate metrics of arthritis severity (Figure 2, red) or other traits related to the humoral response, inflammatory response, or host defense (Figure 2, blue). As predicted from previous MHC congenic studies, none of the arthritis-associated QTL identified in the three B6: $\mathrm{C} 3 \mathrm{H}$ intercrosses identified the $\mathrm{MHC}$ locus on chromosome 17. Interestingly, ankle swelling did associate with this region in a single backcross $(\mathrm{BALB} / \mathrm{c} \times \mathrm{C} 3 \mathrm{H}) \mathrm{F}_{1} \times \mathrm{BALB} / \mathrm{c}$, with a lod score of 3.1, predicting an association with one or more of the numerous class I, class II, or class III genes in this region. Seven of the 23 QTL were reproduced in multiple crosses (Bbaa2, Bbaa6, Bbaa8, Bbaa10, Bbaa12, Bbaa14, and Bbaa15). The Bbaa2 QTL on Chromosome 5 was reproduced in all four intercross experiments, and in every case the arthritis severity originated from the $\mathrm{C} 3 \mathrm{H}$ parental strain. The lod scores identifying Bbaa2 ranged from 3.5 to 10.2 for the four intercross populations, with the 10.2 


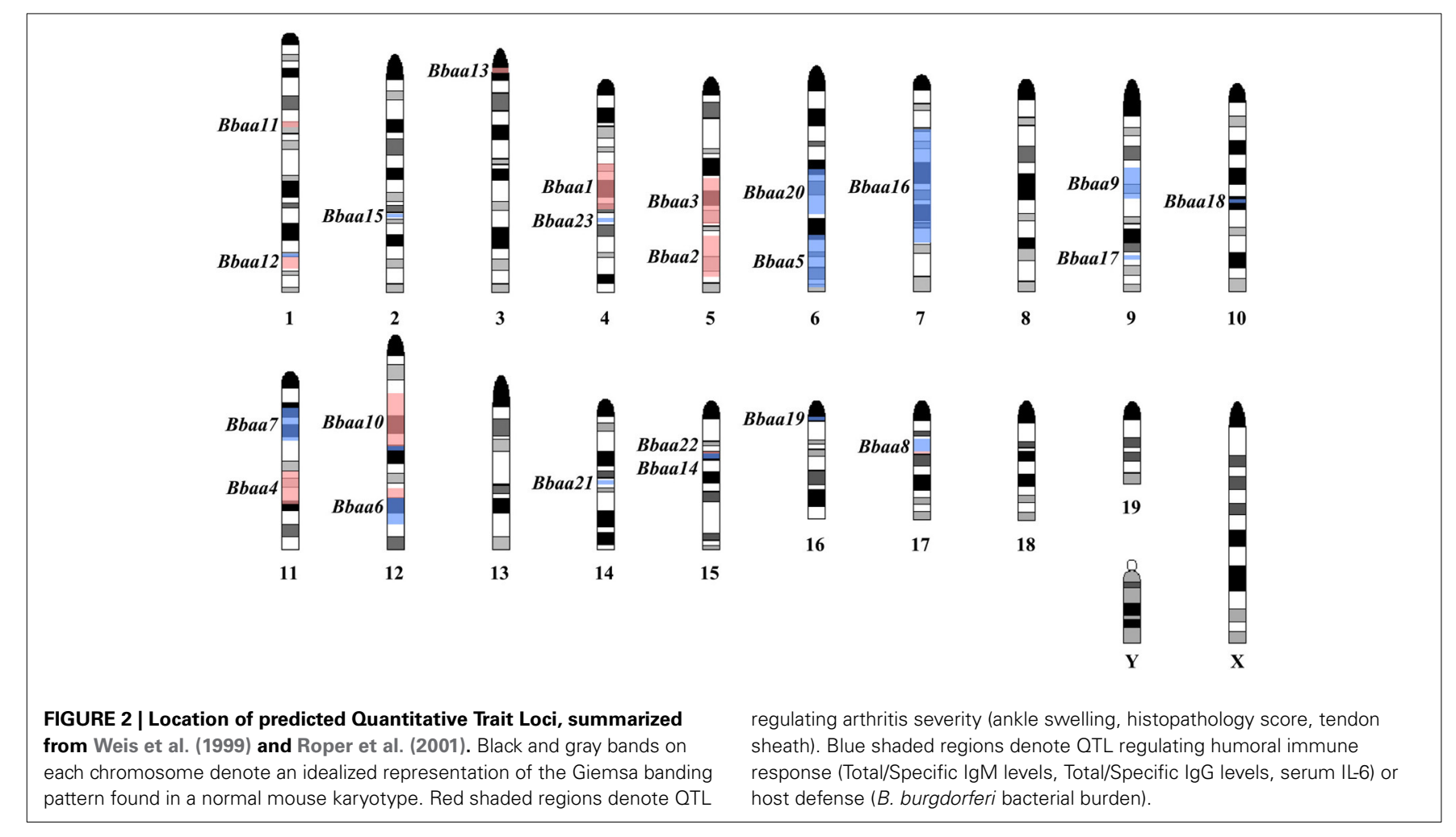

lod value detected in the $(\mathrm{BALB} / \mathrm{c} \times \mathrm{C} 3 \mathrm{H}) \mathrm{F}_{1} \times \mathrm{C} 3 \mathrm{H}$ intercross. This study also predicted that the combined Bbaa2Bbaa 3 locus contains at least four distinct regulatory genes. It is possible that some of these loci may be implicated in other QTL studies, but the use of different inbred strains of mice and the extensive polymorphism of this region of the genome among strains confounds the ability to directly extrapolate between studies (Lindvall et al., 2009).

Based on these and other data, several subsequent studies generated congenic mouse strains to isolate putative regulatory loci in the context of an otherwise uniform genetic background. This laborious and time-intensive process is essential to convert the statistically predicted loci derived from QTL analysis into physical genetic boundaries. Congenic lines can also be used to formally interrogate potential candidate genes, by determining if the phenotype of interest is retained after such a candidate gene is excluded from the congenic interval. The presence of a strongly penetrant phenotype within a congenic interval is a strong predictor of success in further steps of positional cloning.

Subsequent studies used congenic mice based on the initial QTL assignments. Crandall et al. described the phenotype of two $\mathrm{B} 6 \times \mathrm{C} 3 \mathrm{H}$ congenic lines, and the evaluation of a specific candidate gene (Crandall et al., 2005). $\mathrm{B} 6 \times \mathrm{C} 3 \mathrm{H} \mathrm{F}_{1}$ mice were backcrossed seven times onto each parental background, producing reciprocal congenic lines $\mathrm{B} 6 . \mathrm{C} 3 \mathrm{H}-\mathrm{Bbaa2Bbaa3}$ and C3H.B6Bbaa2Bbaa3. Of note, the congenic nomenclature in mice differs from other systems, with the background strain listed first, followed by the donor strain, followed by the introgressed locus (Jackson Laboratory, 2000). Bbaa2Bbaa3 from the C3H donor strain was found to confer increased Lyme arthritis severity on a resistant $\mathrm{B} 6$ background, while $\mathrm{B} 6$ derived Bbaa2Bbaa 3 conferred reduced severity to susceptible $\mathrm{C} 3 \mathrm{H}$ mice, in a reciprocal fashion. This publication also described a polymorphism carried by $\mathrm{C} 3 \mathrm{H}$ mice in the $\mathrm{Ncfl}$ gene, but ruled out this candidate with a variety of studies, including the finding that $\mathrm{B} 6 \mathrm{Ncfl}^{-/-}$mice exhibited no increase in arthritis severity relative to wild type B6 controls.

$\mathrm{Ma}$ et al. reported the generation of additional $\mathrm{B} 6 \mathrm{xC} 3 \mathrm{H}$ reciprocal congenic lines for five intervals identified in the foundational QTL study (Bbaa1, Bbaa2Bbaa3, Bbaa4, and Bbaa6), plus another pair of reciprocal congenic lines for an interval on Chromosome 1 (Bbaa12) (Ma et al., 2009). Through markerassisted selection over the course of seven iterative backcrosses, these intervals were isolated and found to be free of genetic contamination on other chromosomes, with congenic intervals ranging from 25 to 146 megabases in size. Bbaa2Bbaa 3 and $B b a a 4$ were found to reciprocally transfer the ankle swelling and histopathology phenotypes, while the B6 allele of Bbaa6 transferred protection from ankle swelling and histopathology to the $\mathrm{C} 3 \mathrm{H}$ background. Other congenic intervals conferred no change in arthritis severity or gave inconsistent results. This study also demonstrated the added utility of congenic lines as an experimental resource through comparative microarray gene expression profiling.

In the process of further refining these congenic intervals, Bramwell et al. described the implementation of high throughput SNP genotyping and high resolution melting analysis (Bramwell et al., 2012). This improved genotyping methodology took advantage of the recently published Sanger high-resolution sequence of the $\mathrm{C} 3 \mathrm{H}$ mouse, allowing enhanced comparison with the previously published genome of the B6 reference strain (Keane et al., 
2011). The Sanger database revealed the precise location of thousands of SNPs distinguishing $\mathrm{B} 6$ and $\mathrm{C} 3 \mathrm{H}$ genomic sequences within the $20 \mathrm{Mbp}$ Bbaa2 interval, exponentially increasing the ability to discriminate donor sequences and define boundaries of congenic mice, and allowing the genetic composition of the congenic lines to be tested with greater precision (Figure 3). As an added benefit, the screening process was accelerated, helping to reduce expenses by allowing litters to be screened prior to weaning age.

This line of investigation has recently culminated in the identification of the first definitive natural regulator of Lyme arthritis severity in laboratory mice. With further backcrossing and refinement of the B6.C3H-Bbaa 2 congenics, Bramwell et al. describe the generation of 14 new advanced congenics that delimit the boundaries of several regulatory sub-intervals (Figure 4) (Bramwell et al., 2014). Notably, these intervals bear striking resemblance to several of the maximal linkage peaks predicted previously (Roper et al., 2001). One narrow 1.5 Mb C3H-derived interval, surrounding and including the highest peak of linkage predicted by QTL analysis at D5Mit30 on Chromosome 5, was able to independently confer an increased arthritis severity phenotype in the context of a resistant B6 genetic background. Close scrutiny of this interval revealed only a single coding-non-synonymous polymorphism between $\mathrm{B} 6$ and $\mathrm{C} 3 \mathrm{H}$ mice. This point mutation in the lysosomal enzyme beta-Glucuronidase leads to a partially hypomorphic allele $\left(G u s b^{h}\right)$ in the C3H, AKR, and CBA/J inbred strains. Peromyscus mice, which do not exhibit Lyme arthritis but serve as important reservoir hosts for B. burgdorferi in nature, appear to carry the wild-type B6 allele of Gusb (GenBank Accession XM_006971357). The exacerbated Lyme arthritis effect conferred by $G u s b^{h}$ was recapitulated in a spontaneous Gusb mutant mouse line $\left(G u s b^{\text {Null }}\right)$, and transgenic overexpression of wild type $G u s b^{b}$ in $\mathrm{C} 3 \mathrm{H}$ mice $\left(G u s b^{T g}\right)$ profoundly reduced ankle swelling and histopathology. The Gusb ${ }^{h}$ congenic line was further tested in an experimental model of RA,

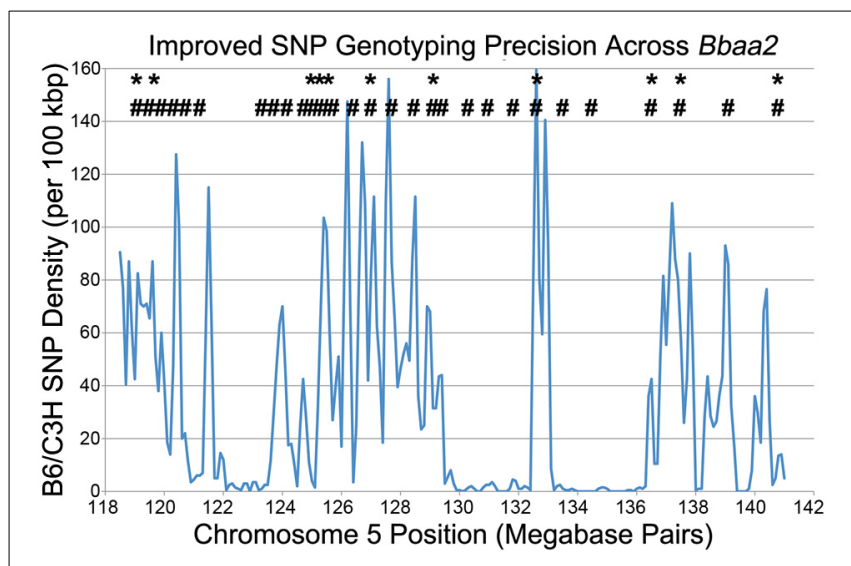

FIGURE 3 | A SNP genotyping methodology improves congenic mapping precision, adapted from Bramwell et al. (2012). * - Location of all 11 microsatellite markers that can differentiate between $\mathrm{B} 6$ and $\mathrm{C} 3 \mathrm{H}$ DNA across Bbaa2. \# - Of the thousands of SNPs available (blue line), the 28 positions that were developed into SNP genotyping assays for improved discrimination of sub-interval congenic lines (Bramwell et al., 2014). the $\mathrm{K} / \mathrm{BxN}$ serum transfer model (Monach et al., 2008). Disease severity in this model is induced by autoantibodies generated against glucose-6-phosphate isomerase, a ubiquitous glycolytic enzyme. Importantly, transfer of this serum induces a joint specific inflammatory arthritis that occurs independently of the MHC haplotype of the recipient and reflects the effector phase of arthritis development. Much greater arthritis severity was observed in $G u s b^{h}$ congenic mice than in wild type B6 control animals, revealing a common mechanism for the pathogenesis of Lyme arthritis and rheumatoid arthritis. Thus, the identification of genes important in Lyme arthritis also illuminated previously unrecognized pathways in RA. This linkage to a gene associated with Sly syndrome, an overt congenital lysosomal storage disease (LSD), strongly implicated a common pathogenic mechanism involving accumulation of undigested glycosaminoglycans (Tomatsu et al., 2009). This possibility was confirmed by detection of pronounced Alcian blue staining of sulfated GAGs in the inflamed joint tissues of B. burgdorferi infected and $\mathrm{K} / \mathrm{BxN}$ treated mice with partial or severe Gusb deficiencies (Figure 5). The association of $G u s b^{h}$ with increased disease severity in both Lymeassociated and rheumatoid arthritis identifies Gusb as a shared immunopathology disease gene (Teuscher, 1985; Sudweeks et al., 1993; Ma et al., 2002).

The novelty of the beta-Glucuronidase polymorphism highlights the power and added value of forward genetic approaches. Gusb is most often cited in the recent scientific literature as a housekeeping gene, primarily used as a reference to study something more interesting, making it a most unlikely candidate for a hypothesis-driven reverse genetics study. Allelic variants of the Gusb gene were found not to be differentially expressed under

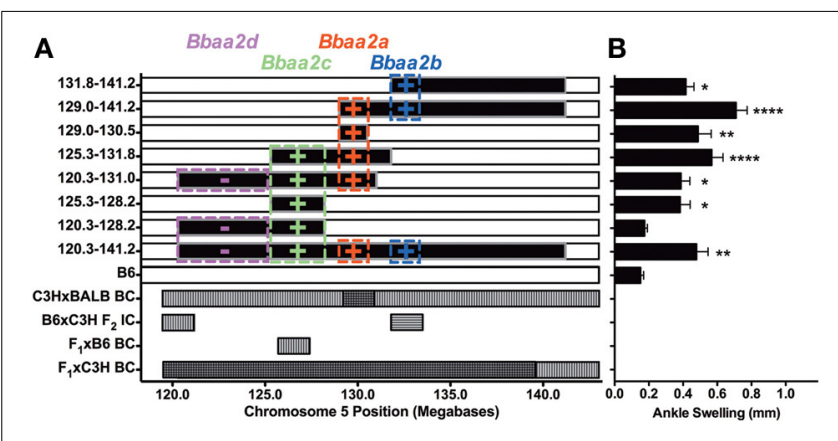

FIGURE 4 | Regulatory loci identified by analysis of advanced congenic lines, modified from Bramwell et al. (2014). (A) Each of the top 8 horizontal bars represents one $\mathrm{B} 6 . \mathrm{C} 3 \mathrm{H}$-Bbaa2 sub-interval congenic mouse line. The horizontal axis represents the position of Bbaa2 on mouse Chromosome 5 (120.3-141.2 Mb). The black portions of each row are derived from the $\mathrm{C} 3 \mathrm{H}$ genetic background and the white portions are derived from the B6 background. Colored boxes indicate the position boundaries and predicted effect (+/-) of multiple regulatory intervals identified by advanced congenic lines. The lower 4 rows indicate arthritis severity QTL intervals predicted by Roper et al. (2001) for the backcross/intercross populations listed on the left vertical axis. Horizontal hatching denotes QTL for ankle swelling, vertical hatching denotes QTL for histopathology or tendon sheath thickening, cross hatching denotes overlap of multiple predicted QTL. (B) Ankle swelling measurements for the eight congenic lines listed in $A$, with significance assessed relative to $B 6$ negative control. ${ }^{*} P<0.05,{ }^{* *} P<0.01,{ }^{* * * *} P<0.0001$. 

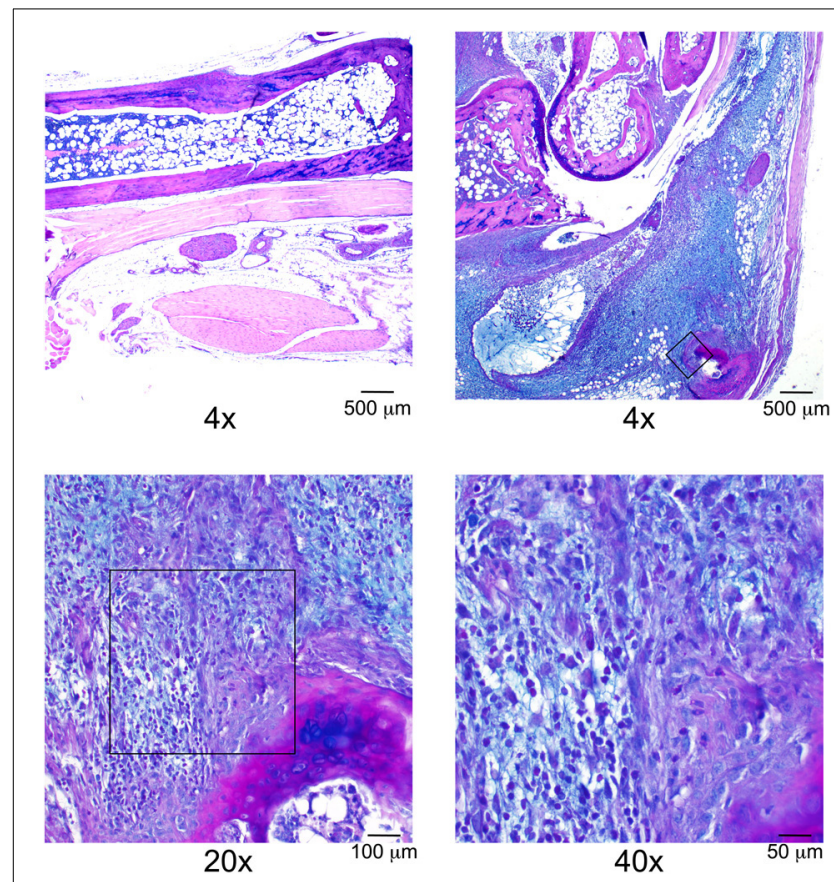

FIGURE 5 | Alcian blue staining reveals excess deposition of GAGs within severely arthritic ankle joints, modified from Bramwell et al. (2014). Top left panel: Ankle joint section from a day $7 \mathrm{~K} / \mathrm{B} \times \mathrm{N}$ treated $\mathrm{B} 6$ mouse at $\times 4$ magnification. Remaining panels: Ankle joint section from a day $7 \mathrm{~K} / \mathrm{B} \times \mathrm{N}$ treated $\mathrm{B} 6 . \mathrm{C} 3 \mathrm{H}-\mathrm{Gusb}^{h}$ mouse. Original magnification $\times 4, \times 20$, and $\times 40$. Scale bars: $500,100,50 \mu \mathrm{m}$, respectively. Boxes on the top right and lower left images indicate the location of the field magnified in subsequent images.

baseline conditions, and no changes in Gusb expression were detected by microarray analysis of joint tissue from naïve and infected C3H and B6 mice (Crandall et al., 2006; Bramwell et al., 2014). Thus, Gusb and other similar genes associated with LSD are not likely to be picked up by a microarray or RNA-Seq study in Lyme arthritis patients. Gusb was also not included in the ImmunoChip used in human RA and juvenile RA studies, because it had not yet been identified as a potential regulator (Eyre et al., 2012; Hinks et al., 2013). As mentioned earlier, recent development of an expanded group of recombinant inbred strains incorporating 8 strains of laboratory and wild mice would appear to be a powerful resource for investigating regulators of Lyme arthritis severity. However, none of the three inbred strains carrying the Gusb $b^{h}$ polymorphism were included in the Collaborative Cross, so it could not have been identified through this approach. Against all odds, the identification of Gusb is a prime example of how relentlessly following the phenotype throughout a process of unbiased genetic refinement can overcome preconception and bias to lead the way to truly novel and unexpected discoveries.

\section{ACKNOWLEDGMENTS}

The mouse chromosomal idiograms used in Figure 2 were derived from images produced by Dr. David Adler (University of Washington). This work was supported by 6133/Arthritis Foundation (to Kenneth K.C. Bramwell) and AR-43521/National
Institute of Arthritis and Musculoskeletal and Skin Diseases (NIAMS) (to Cory Teuscher and Janis J. Weis).

\section{REFERENCES}

Ahlqvist, E., Hultqvist, M., and Holmdahl, R. (2009). The value of animal models in predicting genetic susceptibility to complex diseases such as rheumatoid arthritis. Arthritis Res. Ther. 11, 226. doi: 10.1186/ar2600

Alexopoulou, L., Thomas, V., Schnare, M., Lobet, Y., Anguita, J., Schoen, R. T., et al. (2002). Hyporesponsiveness to vaccination with Borrelia burgdorferi OspA in humans and in TLR1- and TLR2-deficient mice. Nat. Med. 8, 878-884. doi: $10.1038 / \mathrm{nm} 732$

Aliprantis, A. O., Yang, R. B., Mark, M. R., Suggett, S., Devaux, B., Radolf, J. D., et al. (1999). Cell activation and apoptosis by bacterial lipoproteins through Toll-like receptor-2. Science 285, 736-739.

Barthold, S. W., Beck, D. S., Hansen, G. M., Terwilliger, G. A., and Moody, K. D. (1990). Lyme borreliosis in selected strains and ages of laboratory mice. J. Infect. Dis. 162, 133-138.

Barthold, S. W., Sidman, C. L., and Smith, A. L. (1992). Lyme borreliosis in genetically resistant and susceptible mice with severe combined immunodeficiency. Am. J. Trop. Med. Hyg. 47, 605-613.

Bramwell, K. K., Ma, Y., Weis, J. H., Chen, X., Zachary, J. F., Teuscher, C., et al. (2014). Lysosomal beta-glucuronidase regulates Lyme and rheumatoid arthritis severity. J. Clin. Invest. 124, 311-320. doi: 10.1172/JCI72339

Bramwell, K. K., Ma, Y., Weis, J. H., Teuscher, C., and Weis, J. J. (2012). Highthroughput genotyping of advanced congenic lines by high resolution melting analysis for identification of Bbaa2, a QTL controlling Lyme arthritis. BioTechniques 52, 183-190. doi: 10.2144/000113838

Brightbill, H. D., Libraty, D. H., Krutzik, S. R., Yang, R. B., Belisle, J. T., Bleharski, J. R., et al. (1999). Host defense mechanisms triggered by microbial lipoproteins through Toll-like receptors. Science 285, 732-736.

Brown, C. R., and Reiner, S. L. (1999). Genetic control of experimental Lyme arthritis in the absence of specific immunity. Infect. Immun. 67, 1967-1973.

Brown, C. R., and Reiner, S. L. (2000). Genes outside the major histocompatibility complex control resistance and susceptibility to experimental Lyme arthritis. Med. Microbiol. Immunol. 189, 85-90. doi: 10.1007/s004300000044

Bunikis, J., Tsao, J., Luke, C. J., Luna, M. G., Fish, D., and Barbour, A. G. (2004). Borrelia burgdorferi infection in a natural population of Peromyscus Leucopus mice: a longitudinal study in an area where Lyme Borreliosis is highly endemic. J. Infect. Dis. 189, 1515-1523. doi: 10.1086/382594

C.D.C. (2013a). Reported Cases of Lyme Disease by Year, United States, 2003-2012. [Online]. CDC. Available online at: http://www.cdc.gov/lyme/stats/ chartstables/casesbyyear.html (Accessed April 9, 2014).

C.D.C. (2013b). Clinical Manifestations of Confirmed Lyme Disease Cases, United States, 2001-2010 [Online]. CDC. Available online at: http://www.cdc.gov/lyme/ stats/chartstables/casesbysymptom.html (Accessed April 9, 2014).

C.D.C. (2013c). Three sudden cardiac deaths associated with Lyme carditis - United States, November 2012-July 2013. Morb. Mortal. Wkly. Rep. 62, 993-996.

Chen, Y., Perry, D., Boackle, S. A., Sobel, E. S., Molina, H., Croker, B. P., et al. (2005). Several genes contribute to the production of autoreactive B and T cells in the murine lupus susceptibility locus Sle1c. J. Immunol. 175, 1080-1089. doi: 10.4049/jimmunol.175.2.1080

Churchill, G. A., Airey, D. C., Allayee, H., Angel, J. M., Attie, A. D., Beatty, J., et al. (2004). The collaborative cross, a community resource for the genetic analysis of complex traits. Nat. Genet. 36, 1133-1137. doi: 10.1038/ng1104-1133

Crandall, H., Dunn, D. M., Ma, Y., Wooten, R. M., Zachary, J. F., Weis, J. H., et al. (2006). Gene expression profiling reveals unique pathways associated with differential severity of Lyme arthritis. J. Immunol. 177, 7930-7942. doi: 10.4049/jimmunol.177.11.7930

Crandall, H., Ma, Y., Dunn, D. M., Sundsbak, R. S., Zachary, J. F., Olofsson, P., et al. (2005). Bb2Bb3 regulation of murine Lyme arthritis is distinct from Ncfl and independent of the phagocyte nicotinamide adenine dinucleotide phosphate oxidase. Am. J. Pathol. 167, 775-785. doi: 10.1016/S0002-9440(10) 62050-0

Del Rio, R., Noubade, R., Subramanian, M., Saligrama, N., Diehl, S., Rincon, M., et al. (2008). SNPs upstream of the minimal promoter control IL2 expression and are candidates for the autoimmune disease-susceptibility locus Aod2/Idd3/Eae3. Genes Immun. 9, 115-121. doi: 10.1038/sj.gene. 6364455 
Drouin, E. E., Seward, R. J., Strle, K., McHugh, G., Katchar, K., Londono, D., et al. (2013). A novel human autoantigen, endothelial cell growth factor, is a target of $\mathrm{T}$ and $\mathrm{B}$ cell responses in patients with Lyme disease. Arthritis Rheum. 65, 186-196. doi: 10.1002/art.37732

Eyre, S., Bowes, J., Diogo, D., Lee, A., Barton, A., Martin, P., et al. (2012). High-density genetic mapping identifies new susceptibility loci for rheumatoid arthritis. Nat. Genet. 44, 1336-1340. doi: 10.1038/ng.2462

Feng, S., Barthold, S. W., Bockenstedt, L. K., Zaller, D. M., and Fikrig, E. (1995). Lyme disease in human DR4Dw4-transgenic mice. J. Infect. Dis. 172, 286-289.

Garcia-Monco, J. C., and Benach, J. L. (2013). A disconnect between the neurospirochetoses in humans and rodent models of disease. PLoS Pathog. 9:e1003288. doi: 10.1371/journal.ppat.1003288

Glant, T. T., Adarichev, V. A., Nesterovitch, A. B., Szanto, S., Oswald, J. P., Jacobs, J. J., et al. (2004). Disease-associated qualitative and quantitative trait loci in proteoglycan-induced arthritis and collagen-induced arthritis. Am. J. Med. Sci. 327, 188-195. doi: 10.1097/00000441-200404000-00004

Gregory, S. G., Sekhon, M., Schein, J., Zhao, S., Osoegawa, K., Scott, C. E., et al. (2002). A physical map of the mouse genome. Nature 418, 743-750. doi: 10.1038 /nature00957

Hinks, A., Cobb, J., Marion, M. C., Prahalad, S., Sudman, M., Bowes, J., et al. (2013). Dense genotyping of immune-related disease regions identifies 14 new susceptibility loci for juvenile idiopathic arthritis. Nat. Genet. 45, 664-669. doi: 10.1038/ng.2614

Hirschfeld, M., Kirschning, C. J., Schwandner, R., Wesche, H., Weis, J. H., Wooten, R. M., et al. (1999). Cutting edge: inflammatory signaling by Borrelia burgdorferi lipoproteins is mediated by Toll-like receptor 2. J. Immunol. 163, 2382-2386.

Hirschfeld, M., Ma, Y., Weis, J. H., Vogel, S. N., and Weis, J. J. (2000). Cutting edge: repurification of lipopolysaccharide eliminates signaling through both human and murine Toll-like receptor 2. J. Immunol. 165, 618-622. doi: 10.4049/jimmunol.165.2.618

Jackson Laboratory. (2000). Nomenclature for Mouse Strains. [Online]. Available online at: http://jaxmice.jax.org/support/nomenclature/index.html (Accessed April 9, 2014).

Keane, T. M., Goodstadt, L., Danecek, P., White, M. A., Wong, K., Yalcin, B., et al. (2011). Mouse genomic variation and its effect on phenotypes and gene regulation. Nature 477, 289-294. doi: 10.1038/nature10413

Klempner, M. S., Wormser, G. H., Wade, K., Trevino, R. P., Tang, J., Kaslow, R. A., et al. (2005). A case-control study to examine HLA haplotype associations in patients with posttreatment chronic Lyme disease. J. Infect. Dis. 192, 1010-1013. doi: $10.1086 / 432733$

Kuehn, B. M. (2013). CDC estimates 300,000 US cases of Lyme disease annually. JAMA 310, 1110. doi: 10.1001/jama.2013.278331

Kumar, K. R., Li, L., Yan, M., Bhaskarabhatla, M., Mobley, A. B., Nguyen, C., et al. (2006). Regulation of B cell tolerance by the lupus susceptibility gene Lyl08. Science 312, 1665-1669. doi: 10.1126/science.1125893

Lindvall, T., Karlsson, J., Holmdahl, R., and Andersson, A. (2009). Dissection of a locus on mouse chromosome 5 reveals arthritis promoting and inhibitory genes. Arthritis Res. Ther. 11, R10. doi: 10.1186/ar2597

Liu, K., Li, Q. Z., Delgado-Vega, A. M., Abelson, A. K., Sanchez, E., Kelly, J. A., et al. (2009). Kallikrein genes are associated with lupus and glomerular basement membrane-specific antibody-induced nephritis in mice and humans. J. Clin. Invest. 119, 911-923. doi: 10.1172/JCI36728

Ma, R. Z., Gao, J., Meeker, N. D., Fillmore, P. D., Tung, K. S., Watanabe, T., et al. (2002). Identification of Bphs, an autoimmune disease locus, as histamine receptor H1. Science 297, 620-623. doi: 10.1126/science.1072810

Ma, Y., Miller, J. C., Crandall, H., Larsen, E. T., Dunn, D. M., Weiss, R. B., et al. (2009). Interval-specific congenic lines reveal quantitative trait Loci with penetrant Lyme arthritis phenotypes on chromosomes 5, 11, and 12. Infect. Immun. 77, 3302-3311. doi: 10.1128/IAI.00396-09

Meeker, N. D., Hickey, W. F., Korngold, R., Hansen, W. K., Sudweeks, J. D., Wardell, B. B., et al. (1995). Multiple loci govern the bone marrow-derived immunoregulatory mechanism controlling dominant resistance to autoimmune orchitis. Proc. Natl. Acad. Sci. U.S.A. 92, 5684-5688.

Monach, P. A., Mathis, D., and Benoist, C. (2008). The K/BxN arthritis model. Curr. Protoc. Immunol. Chapter 15, Unit 15.22. doi: 10.1002/0471142735.im1522s81

Oosting, M., Ter Hofstede, H., Sturm, P., Adema, G. J., Kullberg, B. J., Van Der Meer, J. W., et al. (2011a). TLR1/TLR2 heterodimers play an important role in the recognition of Borrelia spirochetes. PLOS ONE 6:e25998. doi: 10.1371/journal.pone.0025998
Oosting, M., Ter Hofstede, H., Van De Veerdonk, F. L., Sturm, P., Kullberg, B. J., Van Der Meer, J. W., et al. (2011b). Role of interleukin-23 (IL-23) receptor signaling for IL-17 responses in human Lyme disease. Infect. Immun. 79, 4681-4687. doi: 10.1128/IAI.05242-11

Paterson, A. H., Lander, E. S., Hewitt, J. D., Peterson, S., Lincoln, S. E., and Tanksley, S. D. (1988). Resolution of quantitative traits into Mendelian factors by using a complete linkage map of restriction fragment length polymorphisms. Nature 335, 721-726. doi: 10.1038/335721a0

Radolf, J. D., Caimano, M. J., Stevenson, B., and Hu, L. T. (2012). Of ticks, mice and men: understanding the dual-host lifestyle of Lyme disease spirochaetes. Nat. Rev. Microbiol. 10, 87-99. doi: 10.1038/nrmicro2714

Radolf, J. D., Norgard, M. V., Brandt, M. E., Isaacs, R. D., Thompson, P. A., and Beutler, B. (1991). Lipoproteins of Borrelia burgdorferi and Treponema pallidum activate cachectin/tumor necrosis factor synthesis. Analysis using a CAT reporter construct. J. Immunol. 147, 1968-1974.

Rahman, Z. S., Niu, H., Perry, D., Wakeland, E., Manser, T., and Morel, L. (2007). Expression of the autoimmune Fcgr2b NZW allele fails to be upregulated in germinal center B cells and is associated with increased IgG production. Genes Immun. 8, 604-612. doi: 10.1038/sj.gene.6364423

Risch, N., and Merikangas, K. (1996). The future of genetic studies of complex human diseases. Science 273, 1516-1517.

Roper, R. J., Weis, J. J., McCracken, B. A., Green, C. B., Ma, Y., Weber, K. S., et al. (2001). Genetic control of susceptibility to experimental Lyme arthritis is polygenic and exhibits consistent linkage to multiple loci on chromosome 5 in four independent mouse crosses. Genes Immun. 2, 388-397. doi: 10.1038/sj.gene.6363801

Schaible, U. E., Kramer, M. D., Museteanu, C., Zimmer, G., Mossmann, H., and Simon, M. M. (1989). The severe combined immunodeficiency (scid) mouse. A laboratory model for the analysis of Lyme arthritis and carditis. J. Exp. Med. 170, 1427-1432.

Schroder, N. W., Diterich, I., Zinke, A., Eckert, J., Draing, C., Von Baehr, V., et al. (2005). Heterozygous Arg753Gln polymorphism of human TLR-2 impairs immune activation by Borrelia burgdorferi and protects from late stage Lyme disease. J. Immunol. 175, 2534-2540. doi: 10.4049/jimmunol.175.4.2534

Sellati, T. J., Bouis, D. A., Kitchens, R. L., Darveau, R. P., Pugin, J., Ulevitch, R. J., et al. (1998). Treponema pallidum and Borrelia burgdorferi lipoproteins and synthetic lipopeptides activate monocytic cells via a CD14-dependent pathway distinct from that used by lipopolysaccharide. J. Immunol. 160, 5455-5464.

Spach, K. M., Case, L. K., Noubade, R., Petersen, C. B., McElvany, B., Zalik, N., et al. (2010). Multiple linked quantitative trait loci within the Tmevd2/Eae3 interval control the severity of experimental allergic encephalomyelitis in DBA/2J mice. Genes Immun. 11, 649-659. doi: 10.1038/gene.2010.40

Spach, K. M., Noubade, R., McElvany, B., Hickey, W. F., Blankenhorn, E. P., and Teuscher, C. (2009). A single nucleotide polymorphism in Tyk2 controls susceptibility to experimental allergic encephalomyelitis. J. Immunol. 182, 7776-7783. doi: 10.4049/jimmunol.0900142

Steere, A. C., and Glickstein, L. (2004). Elucidation of Lyme arthritis. Nat. Rev. Immunol. 4, 143-152. doi: 10.1038/nri1267

Steere, A. C., Dwyer, E., and Winchester, R. (1990). Association of chronic Lyme arthritis with HLA-DR4 and HLA-DR2 alleles. N. Engl. J. Med. 323, 219-223. doi: 10.1056/NEJM199007263230402

Steere, A. C., Klitz, W., Drouin, E. E., Falk, B. A., Kwok, W. W., Nepom, G. T., et al. (2006). Antibiotic-refractory Lyme arthritis is associated with HLA-DR molecules that bind a Borrelia burgdorferi peptide. J. Exp. Med. 203, 961-971. doi: 10.1084/jem.20052471

Strle, K., Shin, J. J., Glickstein, L. J., and Steere, A. C. (2012). Association of a Tolllike receptor 1 polymorphism with heightened Th1 inflammatory responses and antibiotic-refractory Lyme arthritis. Arthritis Rheum. 64, 1497-1507. doi: 10.1002/art.34383

Sudweeks, J. D., Todd, J. A., Blankenhorn, E. P., Wardell, B. B., Woodward, S. R., Meeker, N. D., et al. (1993). Locus controlling Bordetella pertussis-induced histamine sensitization (Bphs), an autoimmune disease-susceptibility gene, maps distal to T-cell receptor beta-chain gene on mouse chromosome 6. Proc. Natl. Acad. Sci. U.S.A. 90, 3700-3704.

Tchepeleva, S. N., Thurman, J. M., Ruff, K., Perkins, S. J., Morel, L., and Boackle, S. A. (2010). An allelic variant of Crry in the murine Sle1c lupus susceptibility interval is not impaired in its ability to regulate complement activation. J. Immunol. 185, 2331-2339. doi: 10.4049/jimmunol.1000783 
Teuscher, C. (1985). Experimental allergic orchitis in mice. II. Association of disease susceptibility with the locus controlling Bordetella pertussis-induced sensitivity to histamine. Immunogenetics 22, 417-425.

Teuscher, C., Hickey, W. F., Grafer, C. M., and Tung, K. S. (1998). A common immunoregulatory locus controls susceptibility to actively induced experimental allergic encephalomyelitis and experimental allergic orchitis in BALB/c mice. J. Immunol. 160, 2751-2756.

Teuscher, C., Rhein, D. M., Livingstone, K. D., Paynter, R. A., Doerge, R. W., Nicholson, S. M., et al. (1997). Evidence that Tmevd2 and eae3 may represent either a common locus or members of a gene complex controlling susceptibility to immunologically mediated demyelination in mice. J. Immunol. 159, 4930-4934.

Teuscher, C., Wardell, B. B., Lunceford, J. K., Michael, S. D., and Tung, K. S. (1996). Aod2, the locus controlling development of atrophy in neonatal thymectomyinduced autoimmune ovarian dysgenesis, co-localizes with $I l 2, F g f b$, and $I d d 3$. J. Exp. Med. 183, 631-637.

Todd, J. A., Aitman, T. J., Cornall, R. J., Ghosh, S., Hall, J. R., Hearne, C. M., et al. (1991). Genetic analysis of autoimmune type 1 diabetes mellitus in mice. Nature 351, 542-547. doi: 10.1038/351542a0

Tomatsu, S., Montano, A. M., Dung, V. C., Grubb, J. H., and Sly, W. S. (2009). Mutations and polymorphisms in GUSB gene in mucopolysaccharidosis VII (Sly Syndrome). Hum. Mutat. 30, 511-519. doi: 10.1002/humu.20828

Tschirren, B., Andersson, M., Scherman, K., Westerdahl, H., Mittl, P. R., and Raberg, L. (2013). Polymorphisms at the innate immune receptor TLR2 are associated with Borrelia infection in a wild rodent population. Proc. Biol. Sci. 280, 20130364. doi: 10.1098/rspb.2013.0364

Weis, J. J., McCracken, B. A., Ma, Y., Fairbairn, D., Roper, R. J., Morrison, T. B., et al. (1999). Identification of quantitative trait loci governing arthritis severity and humoral responses in the murine model of Lyme disease. J. Immunol. 162, 948-956.

Wicker, L. S., Clark, J., Fraser, H. I., Garner, V. E., Gonzalez-Munoz, A., Healy, B., et al. (2005). Type 1 diabetes genes and pathways shared by humans and NOD mice. J. Autoimmun. 25 (Suppl.), 29-33. doi: 10.1016/j.jaut.2005.09.009
Wooley, P. H., Luthra, H. S., Stuart, J. M., and David, C. S. (1981). Type II collageninduced arthritis in mice. I. Major histocompatibility complex (I region) linkage and antibody correlates. J. Exp. Med. 154, 688-700.

Wooten, R. M., Modur, V. R., McIntyre, T. M., and Weis, J. J. (1996). Borrelia burgdorferi outer membrane protein A induces nuclear translocation of nuclear factor-kappa B and inflammatory activation in human endothelial cells. J. Immunol. 157, 4584-4590.

Wormser, G. P., Dattwyler, R. J., Shapiro, E. D., Halperin, J. J., Steere, A. C., Klempner, M. S., et al. (2006). The clinical assessment, treatment, and prevention of Lyme disease, human granulocytic anaplasmosis, and babesiosis: clinical practice guidelines by the Infectious Diseases Society of America. Clin. Infect. Dis. 43, 1089-1134. doi: 10.1086/508667

Yang, L., Ma, Y., Schoenfeld, R., Griffiths, M., Eichwald, E., Araneo, B., et al. (1992). Evidence for B-lymphocyte mitogen activity in Borrelia burgdorferi-infected mice. Infect. Immun. 60, 3033-3041.

Conflict of Interest Statement: The authors declare that the research was conducted in the absence of any commercial or financial relationships that could be construed as a potential conflict of interest.

Received: 30 January 2014; paper pending published: 28 March 2014; accepted: 20 May 2014; published online: 05 June 2014.

Citation: Bramwell KK, Teuscher C and Weis JJ (2014) Forward genetic approaches for elucidation of novel regulators of Lyme arthritis severity. Front. Cell. Infect. Microbiol. 4:76. doi: 10.3389/fcimb.2014.00076

This article was submitted to the journal Frontiers in Cellular and Infection Microbiology.

Copyright (C) 2014 Bramwell, Teuscher and Weis. This is an open-access article distributed under the terms of the Creative Commons Attribution License (CC BY). The use, distribution or reproduction in other forums is permitted, provided the original author(s) or licensor are credited and that the original publication in this journal is cited, in accordance with accepted academic practice. No use, distribution or reproduction is permitted which does not comply with these terms. 